\title{
Valproic Acid: Second Generation
}

\author{
Meir Bialer* and Boris Yagen ${ }^{\dagger}$ \\ *Department of Pharmaceutics and ${ }^{\dagger}$ Department of Medicinal Chemistry and Natural Products, School of Pharmacy, Faculty of \\ Medicine, The Hebrew University of Jerusalem, Jerusalem, Israel, Affiliated with the David R. Bloom Centre for Pharmacy, The \\ Hebrew University of Jerusalem
}

\begin{abstract}
Summary: The manuscript focuses on structure-activity relationship studies of CNS-active compounds derived from valproic acid (VPA) that have the potential to become secondgeneration VPA drugs. Valproic acid is one of the four most widely prescribed antiepileptic drugs (AEDs) and is effective (and regularly approved) in migraine prophylaxis and in the treatment of bipolar disorders. Valproic acid is also currently undergoing clinical trials in cancer patients. Valproic acid is the least potent of the established AEDs and its use is limited by two rare but potentially life-threatening side effects, teratogenicity and hepatotoxicity. Because AEDs treat the symptoms
\end{abstract}

(seizure) and not the cause of epilepsy, epileptic patients need to take AEDs for a long period of time. Consequently, there is a substantial need to develop better and safer AEDs. To become a successful second-generation VPA, the new drug should possess the following characteristics: broad-spectrum antiepileptic activity, better potency than VPA, lack of teratogenicity and hepatotoxicity, and a favorable pharmacokinetic profile compared with VPA including a low potential for drug interactions. Key Words: Valproic acid, analogs and derivatives of valproic acid, second generation to valproic acid drugs, antiepileptics and CNS drugs.

\section{INTRODUCTION}

This review aims to provide insight into current developments in the search for second-generation VPA drugs based on structure-activity relationship (SAR) studies. In the last decade, 10 new antiepileptic drugs (AEDs) that offer appreciable advantages in terms of favorable pharmacokinetics, improved tolerability, and lower potential for drug interactions have been introduced. ${ }^{1-5}$ The availability of both old and new AEDs with various activity spectra and different tolerability profiles enables clinicians to better tailor drug choice to the characteristics of the individual patient. ${ }^{3}$ However, the new AEDs developed so far are not completely effective, because altogether they result in a seizure-free status in no more than $15-20 \%$ of previously refractory patients. ${ }^{3}$ Thus, in spite of the large therapeutic range of old and new AEDs, about $30 \%$ of epileptic patients are still not seizure-free and a substantial need remains to develop new AEDs. ${ }^{4,5}$

Table 1 lists the new AEDs in development. Most of

Address correspondence and requests for reprints to: Prof. Meir Bialer, Department of Pharmaceutics, School of Pharmacy, Faculty of Medicine, The Hebrew University of Jerusalem, P.O.B. 12065, Ein Karem, Jerusalem 91120, Israel; E-mail: bialer@md.huji.ac.il. them are currently in clinical trials. ${ }^{1,}$ 4-8 The compounds presented in Table 1 can be divided into two categories: 1) new CNS-active molecules (e.g., lacosamide, retigabine and rufinamide); and 2) derivatives of existing CNS drugs being developed as second generation to existing AEDs, such as carbamazepine, valproic acid (VPA), pregabalin, felbamate, and levetiracetam. While most of the new AEDs were developed empirically, and in many cases serendipitously, the second generation of existing AEDs were designed to widen their CNS activity, as well as to improve their efficacy, safety, tolerability, and pharmacokinetic profile.

The following VPA analogs and derivatives are critically reviewed in this manuscript (FIGS. 1 and 2): valrocemide; isovaleramide (NPS 1776); valnoctamide (VCD); propylisopropylacetamide (PID); 2,2,3,3-teteramethylcylcopropylcarboxylic acid (TMCA), a cyclopropyl analogue of VPA and its amide derivatives: N-methyl2,2,3,3-tetramethylcyclopropanecarboxamide (MTMCD) and 2,2,3,3-tetramethylcyclopropanecarbonyl urea (TMCU). The PID, MTMCD, and TMCU are currently in the preclinical stage, and have a promising potential to become new antiepileptics and CNS drugs as a second generation to VPA. ${ }^{6-8}$ The design of these drugs was motivated by pharmacokinetic and pharmacodynamic principles aiming to: 1) enhance brain penetration; 
TABLE 1. New Antiepileptic Drugs (AEDs) That Are in Development

\begin{tabular}{lc}
\hline \multicolumn{1}{c}{ Drug } & Phase of Development \\
\hline Brivaracetam & I \\
Eslicarbazepine acetate & III \\
Fluorofelbamate & I \\
Ganaxolone & II \\
Huperzine & I \\
JZP-4 & I \\
Lacosamide & II \\
NPS 1776 & II \\
NS1209 & II \\
PID & Preclinical \\
Retigabine & II \\
Rufinamide & III \\
RWJ-333369 & II/III \\
Seletracetam & I \\
Stiripentol & III \\
Talampanel & II \\
Valnoctamide & II (Bipolar disorder) \\
Valrocemide & II \\
XP 13512 & II \\
\hline
\end{tabular}

PID $=$ propylisopropylacetamide.

2) improve anticonvulsant and CNS potency compared with VPA; 3) avoid teratogenicity; and/or d) circumvent the formation of toxic metabolites responsible for VPAassociated hepatotoxicity.

In addition to the above scientific and clinical rationale, there is also a strong economic incentive for the development of second-generation VPA drugs. With worldwide annual sales exceeding $\$ 1$ billion, VPA is a particularly attractive target for further development. ${ }^{4,6}$

\section{THE SECOND GENERATION TO VALPROIC ACID (VPA)}

Valproic acid, one of the established AEDs, is in animal models the least potent of the major AEDs. However, due to its wide spectrum of antiepileptic activity, VPA is the most prescribed AED. ${ }^{9,10}$ Valproic acid is also an effective (and FDA-approved) drug in migraine prophylaxis and in the treatment of bipolar disorder. ${ }^{9-12}$

Valproic acid is a simple molecule (isooctanoic acid) ${ }^{7,12,13}$ and, thus, a useful, cheap, and readily available starting material for synthesizing an array of derivatives that can become CNS-active follow-up compounds to VPA. We believe that novel chemical modifications and further development of specific VPA analogues and derivatives will show promising potential in the areas of epilepsy, pain, bipolar disorder, and other related neurological diseases. As VPA is the least potent among the established AEDs, it is possible to develop VPA analogues that will be significantly more potent than the parent compound and will also be nonteratogenic.

There are numerous reports defining the strict struc- tural requirements for the teratogenicity of VPA and its structurally related compounds. ${ }^{6,7,14}$ Structure-activity relationship studies conducted in mice strains prone to VPA-associated teratogenicity indicate that to be teratogenic, and to cause neural tube defects in mice embryos, VPA analogues and derivatives should contain a tertiary carbon bound to a carboxylic group, a hydrogen atom, and two alkyl chains. ${ }^{14-16}$ A VPA derivative lacking any one of these structural requirements has the potential to become a nonteratogenic entity. ${ }^{6,74-16}$ For example, the corresponding CNS-active amide of VPA valpromide (VPD) that has a carboxamide moiety instead of a carboxylic group is not teratogenic. ${ }^{17}$ Similarly, the active metabolite of VPA is 2-ene-VPA, which does not have an $\alpha$-hydrogen to the carboxylic moiety, is also nonteratogenic. ${ }^{17}$ Valpromide and 2-ene-VPA are potent anticonvulsant compounds that may represent a novel type of second-generation VPA drug. However, as their fraction metabolized to VPA in humans is greater than $90 \%$ (for VPD) and approximately 20\% (for 2-ene-VPA), their lack of teratogenicity does not offer a clinical advantage over VPA. ${ }^{6}$

Unlike teratogenicity, the current thinking on VPAinduced hepatotoxicity (microvesicular steatosis) is that it is not caused by the parent compound but primarily by VPA metabolite(s) with a terminal double-bond: 4-eneVPA and 2,4-diene-VPA. These metabolites are further biotransformed to chemically reactive intermediates that bind to cellular macromolecules and enzymes involved in the metabolism of fatty acids. The first step in this cascade is the formation of an acyl-coenzyme A (CoA) thioester leading to depletion of $\mathrm{CoA}$ in the liver and, consequently, to hepatotoxicity. ${ }^{18-21}$ Designing substituted aliphatic and alicyclic VPA analogues and $\alpha$ and $\beta$ substituted VPA derivatives (amides) to block the formation of these two metabolites should prevent, or at least minimize, the VPA-induced hepatotoxicity. ${ }^{22-24}$

Structure-activity relationship studies mapped the structural elements of the VPA molecules responsible for the anticonvulsant activity. ${ }^{25}$ Subsequent studies showed that constitutional isomers of VPA, such as valnoctic acid (VCA), propylisopropyl acetic acid (PIA), or diisopropylacetic acid (DIA), were less active as anticonvulsants than VPA. However, their respective corresponding amides (FIG. 2), VCD, propylisopropylacetamide (PID), and diisopropylacetamide (DID), are more potent than VPA. ${ }^{26,27}$ Unlike valproyl esters, VPA amide derivatives act as drugs on their own and not as prodrugs to their corresponding acids. ${ }^{6,28}$ Recent SAR data indicate that a pharmacokinetic-based design is an attractive and feasible approach for the development of nonteratogenic and nonhepatotoxic CNS-active second generation to VPA drugs. ${ }^{6-8}$

Currently, the leading compounds that are second generation to VPA can be divided into three groups (FIGS. 

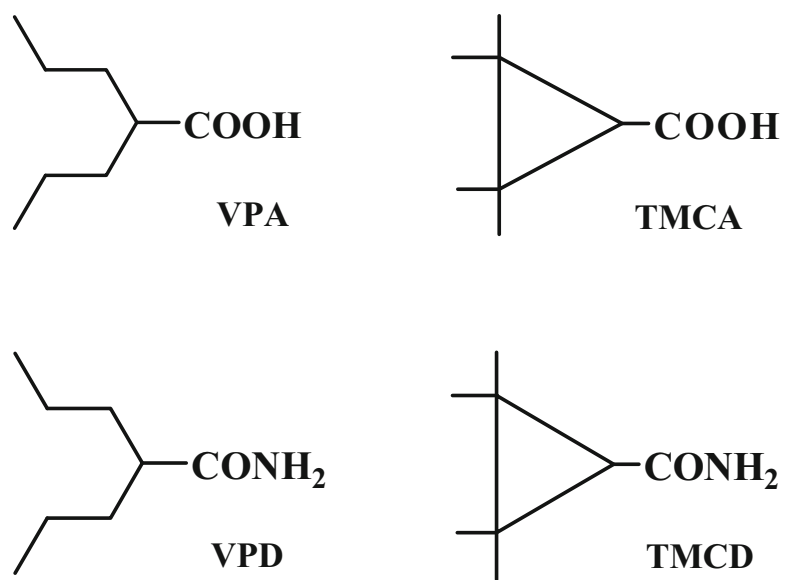

FIG. 1. Valproic acid (VPA), its cyclopropyl analogue 2,2,3,3tetramethylcyclopropanecarboxylic acid (TMCA) and their corresponding amides valpromide (VPD) and 2,2,3,3-tetramethylcyclopropanecarboxamide (TMCD).

$1,2$, and 3$)^{6,7}: 1$ ) alkyl analogues of VPA and their amide derivatives, including chiral and achiral constitutional isomers of VPD (FIG. 2); 2) amide derivatives of TMCA, a cyclopropyl analogue of VPA (FIG. 2); and 3) conjugation products between VPA and neuroinhibitory amino acids: GABA, glycine, taurine, and their corresponding amides (FIG. 3). Each group is discussed below.

\section{ALKYL ANALOGUES OF VPA AND THEIR AMIDE DERIVATIVES}

Valpromide (FIG. 1), the corresponding amide of VPA, was approved as an antipsychotic and an AED in several European countries. ${ }^{29}$ Although VPD is three to five times more potent than VPA and is not teratogenic in animal models, in humans, unlike in mice, rats, or dogs, it serves as a prodrug to VPA. Therefore, its improved anticonvulsant activity and lack of teratogenicity in animal models does not apply to humans. ${ }^{6-8,29}$

Consequently, after a series of structure-pharmacokinetic-pharmacodynamic relationship studies, three CNS-active constitutional isomers of VPD, VCD, propylisopropyl acetamide (PID), and diisopropyl acetamide (DID), have emerged as lead compounds (FIG. 2). ${ }^{6-8,22,30-33}$ The VCD, PID, and DID are more potent as anticonvulsants than their corresponding acids, and act in mice, rats, or dogs as drugs on their own and not as prodrugs to their corresponding acids. ${ }^{26,27}$ PID and VCD are CNS-active nonteratogenic chiral constitutional isomers of VPD that are more potent than VPA and have shown stereoselective pharmacokinetics and pharmacodynamics (anticonvulsant activity). ${ }^{22,30-32}$ The (R)-PID was more potent as an anticonvulsant than (S)-PID and (2S,3S)-VCD was more potent than $(2 \mathrm{R}, 3 \mathrm{~S})-\mathrm{VCD}{ }^{22,31} \mathrm{PID}$ and VCD (racemate and individual stereoisomers) were also very
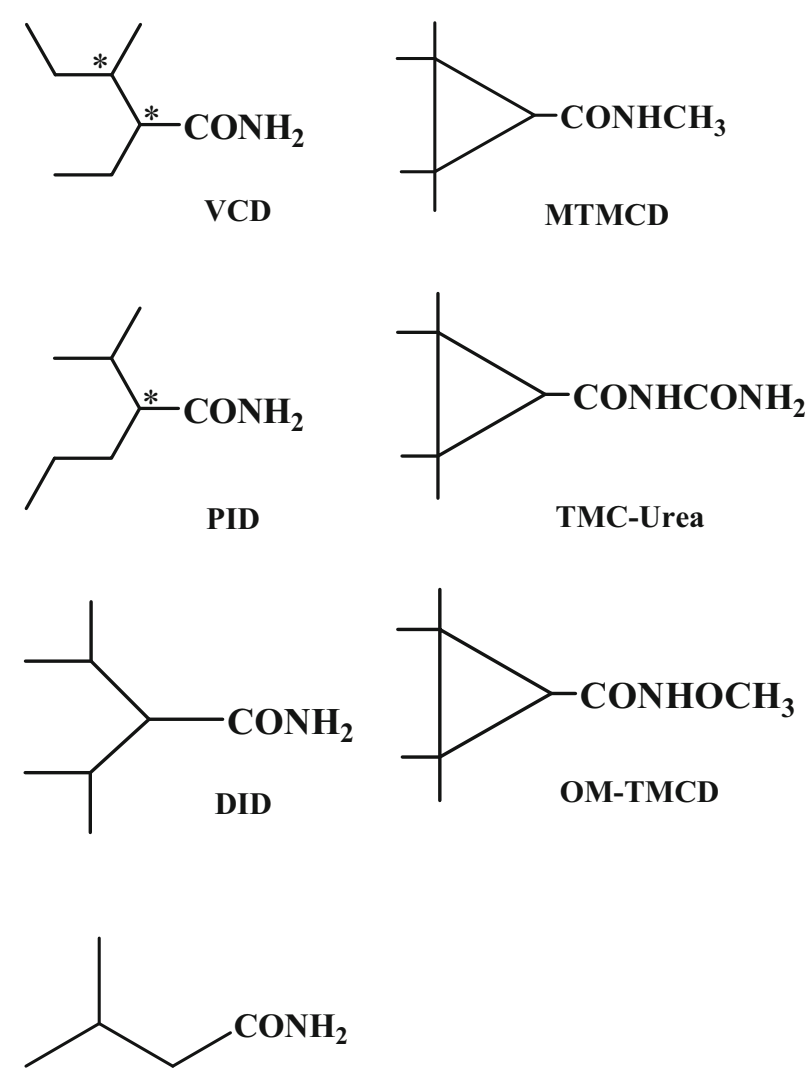

NPS 1776

FIG. 2. Chemical structures of CNS-active amides of valproic acid (VPA) analogues with the potential to become second-generation VPA drugs. Valnoctamide (VCD), propylisopropylacetamide (PID), di-isopropylacetamide (DID), N-methyl-2,2,3,3-tetramethylcylclopropanecarboxamide (MTMCD), 2,2,3,3-tetramethylcyclopropylcarbonyl urea (TMC-urea), N-methoxy-2,2,3,3-tetramethylcylclopropanecarbonylurea (OM-TMCD), and isovaleramide (NPS 1776). ${ }^{*}$ Indicates the chiral center.

active in two animal models of difficult-to-treat seizures, namely the hippocampal kindled rat model and the $6 \mathrm{~Hz}$ psychomotor seizure model in mice, thus suggesting that these amides could be useful in therapy-resistant epileptic patients. ${ }^{22,30,31}$ Racemic VCD demonstrated antiallodynic activity similar to that of
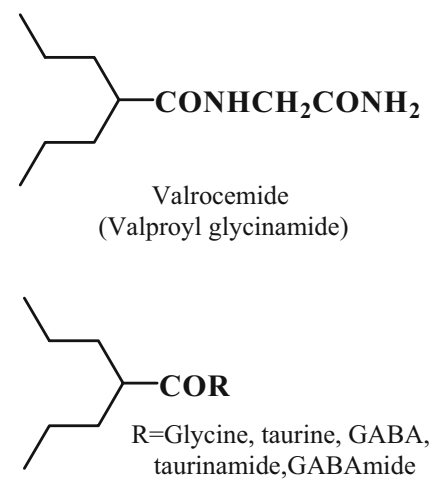

FIG. 3. Valrocemide and conjugation products between valproic acid and neuroinhibitory amino acids and their corresponding amides. 

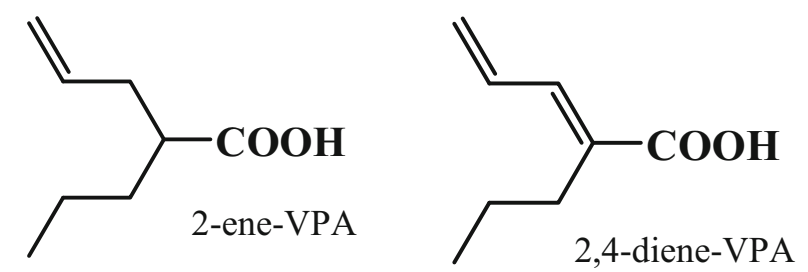

FIG. 4. Hepatotoxic metabolites.

gabapentin in the rat spinal nerve ligation model for neuropathic pain. ${ }^{33}$ A recent clinical trial showed that VCD (racemate, Fig. 2), previously utilized in Europe as an anxiolytic agent (Nirvanil; Ono Pharmaceutical, Co., Ltd., Osaka, Japan), has activity as an add-on drug to risperidone in patients with bipolar disorder. ${ }^{34}$

Isovaleramide (NPS 1776) (FIG. 2) is an amide derivative of isovaleric acid, a VPA analogue characterized by a broad spectrum of anticonvulsant activity in animal models. ${ }^{1}$ Overall it appears similar to VPA but is weaker in potency in animal models. ${ }^{8}$ Isovaleramide has linear pharmacokinetics at a dose range of 100-1600 $\mathrm{mg}$ with complete oral absorption and a short half-life of $2.5 \mathrm{~h} .{ }^{1}$ The short half-life is a major concern and is because NPS-1776 has in its chemical structure three carbons less than VPA (five carbons versus eight). Due to its short half-life, sustained release prototype formulations of the drug have been developed and evaluated in healthy subjects. ${ }^{1}$ Isovaleramide does not inhibit any of the major human cytochromes (P450; CYPs) and, therefore, may not be involved in inhibitory drug interactions. Phase I clinical trials in healthy subjects have indicated that the drug is safe and well tolerated; however, ${ }^{1,8}$ so far no clinical trials in epileptic patients have been reported.

Eadie recently proposed that the herbal remedy valerian, an extract of Valeriana officinalis that contains an array of short-chain fatty acids, is the first antiepileptic drug that is active as a result of isovaleric acid. ${ }^{35}$ Isovaleric acid has an undesirable odor and taste, but its corresponding amide isovaleramide does not. ${ }^{8,35}$ Indeed, NPS 1776 has reached phase I clinical trials, ${ }^{1}$ although its future development is unclear at present.

\section{DERIVATIVES OF TMCA}

Tetramethylcyclopropanecarboxylic acid (FIG. 1) is a cyclopropyl analogue of VPA with two quaternary carbons at the $\beta$-position to the carboxylic group. It has been demonstrated that VPA-associated hepatotoxicity is caused by a metabolite(s) with a terminal double bond (4-ene-VPA and 2,4-diene-VPA; FIG. 4). The TMCA cannot be biotransformed to hepatotoxic metabolites with a terminal double bond $\left(\Delta^{4}\right)$, analogous to 4-eneVPA. ${ }^{6-8,36}$

The TMCA showed low anticonvulsant activity and did not demonstrate separation between its anticonvulsant ac- tivity and neurotoxicity in rodents. ${ }^{24}$ However, the amide derivatives of TMCA, 2,2,3,3-tetramethylcyclopropanecarboxamide (TMCD; FIG. 1), N-methyl-TMCD (MTMCD), and 2,2,3,3-tetramethylcyclopropylcarbonylurea (TMCU; FIG. 2), all possess anticonvulsant activity in rodent models such as the maximal electroshock (MES) and subcutaneous metrazole (scMet) tests with protective indexes improved over that of VPA. .-8,36,37 $^{-1}$

Structure-activity relationship studies of numerous amide derivatives of TMCA determined the structural requirements for anticonvulsant activity in this series of amides. The TMCU and MTMCD (FIG. 2) have emerged as the most promising compounds from this group. $^{23,37,38}$ The MTMCD possesses a broad anticonvulsant spectrum of activity, including efficacy in models of difficult-to-treat seizures, such as those of the hippocampally kindled rat and the $6 \mathrm{~Hz}$ models. ${ }^{37}$ The TMCU was the most potent compound in the rat-MES test $\left(\mathrm{ED}_{50}=29 \mathrm{mg} / \mathrm{kg}\right)$, with a protective index $\left(\mathrm{TD}_{50^{-}}\right.$ to-ED ${ }_{50}$ ratio) of 18.5 in the MES test, compared with 1.6 for VPA. ${ }^{23,38,39}$ Furthermore, both MTMCD and TMCU are nonteratogenic and nonhepatotoxic. ${ }^{37,40}$

We recently discovered that MTMCD and TMCU both possess an antiallodynic activity in the rat spinal nerve ligation (SNL) model for neuropathic pain, and are about 7 and 1.5 times more potent than VPA, respectively. ${ }^{24}$ The MTMCD in the SNL model was equipotent to gabapentin, currently the leading AED in neuropathic pain treatment. ${ }^{24}$ In another study, MTMCD was found to be equipotent to VPA and lithium in various models for bipolar disorders. ${ }^{41}$ Thus, the 2,2,3,3-tetramethylcyclopropane amides MTMCD and TMCU have the potential to become new antiepileptics and CNS drugs as the second generation of VPA.

Another interesting amide derivative of TMCA is Nmethoxy-TMCD (OM-TMCD; FIG. 2), which demonstrated a broad spectrum of anticonvulsant activity and showed better potency than VPA in the MES and scMet tests as well as in the hippocampally kindled rat model. ${ }^{42}$ Teratogenicity studies performed in a SWV/Fnn-mouse model for a VPA-induced exencephaly showed that, on an equimolar basis, OM-TMCD possesses the similar fetal toxicity and ability to induce neural tube defects (NTDs) as VPA, but because OM-TMCD is a much more potent anticonvulsant, its activity-exencephalyformation ratio appears to be much more beneficial than that of VPA. In addition, in the well established Naval Medical Research Institute-mouse model for VPA-induced NTDs, OM-TMCD, administered subcutaneously at $3.6 \mathrm{mmol} / \mathrm{kg}(664 \mathrm{mg} / \mathrm{kg})$ dose, was not lethal to the dams and did not produce even a single case of exencephaly, compared with the same dose of VPA, which induced exencephaly in $27 \%$ of the fetuses. ${ }^{40}$ These results indicate that OM-TMCD, in contrast to VPA, has a lower potential to induce NTDs. The OM-TMCD 
showed a beneficial pharmacokinetic profile in rats; having a high oral bioavailability of $75 \%$ and satisfactory values of clearance and volume of distribution. ${ }^{42}$ These results support further studies to fully characterize the therapeutic potential of OM-TMCD.

\section{CONJUGATION PRODUCTS OF VALPROIC ACID AND NEUROINHIBITORY AMINO ACIDS}

GABA, glycine, and taurine are the major neuroinhibitory transmitters found in the brain. These acids were conjugated to VPA and found to be inactive as anticonvulsants. ${ }^{43}$ Valproyl taurinamide, the conjugation product of VPA and taurinamide, was recently found to possess potent anticonvulsant activity, ${ }^{44}$ although it was not superior to valproyl glycinamide in anticonvulsant activity and lack of teratogenicity. ${ }^{44}$ Valrocemide (valproyl glycinamide; FIG. 3), the conjugation product of VPA and glycinamide, is currently in phase II clinical trials. ${ }^{1,6-8,45,46}$ The design of valrocemide was motivated by pharmacokinetic considerations whose goals were the following: 1) to enhance brain penetration of a glycine derivative; and 2) to utilize the glycinamideto-glycine biotransformation to a known VPA metabolite valproyl glycine as a major hydrolytic nonoxidative metabolic pathway. ${ }^{1,6-8,46}$ In addition to showing antiepileptic activity, valrocemide demonstrated activity in animal models for neuropathic pain and bipolar disorder. ${ }^{1,47,48}$

In humans, valrocemide exhibits linear pharmacokinetics after single oral doses ranging from 250 to 4000 $\mathrm{mg}$, and multiple doses ranging from 250 to $1000 \mathrm{mg}$ three times daily. ${ }^{1}$ Valrocemide's oral clearance and half-life range from 5-7 L/h and 6.4-9.4 h, respectively. About $10-20 \%$ of a valrocemide oral dose is excreted unchanged in the urine and $40 \%$ of the dose is excreted in the urine as valproyl glycine. Overall, the renal clearance of the unchanged drug and the formation clearance of valproyl glycine account for $57 \%$ to $75 \%$ of the oral clearance of valrocemide. Valproic acid is a minor metabolite in humans, and it has been estimated that the fraction of valrocemide metabolized to VPA is about 4-6\% . ${ }^{1,47}$ Preliminary results suggest that patients comedicated with enzyme-inducing AEDs exhibit moderately higher valrocemide clearance and shorter half-lives compared with healthy volunteers or patients not receiving enzyme inducers. ${ }^{1,47}$ Metabolic studies in human liver microsomes have shown that valrocemide and its metabolite valproyl glycine have no inhibiting activity on cytochromes CYP1A2, CYP2A6, CYP2C9, CYP2C19, CYP2D6, CYP2E2, CYP3A4, or epoxide hydrolase. ${ }^{1,47}$

In preliminary open-label adjunctive therapy studies in patients with epilepsy, valrocemide has been found to be well tolerated at maintenance dosages up to $2000 \mathrm{mg}$ twice daily. ${ }^{1,47}$ Most adverse events were mild to moderate and affected the CNS or the gastrointestinal tract. Many patients reported lower seizure frequency during valrocemide treatment compared with baseline. Placebocontrolled randomized trials are planned to confirm these findings. In July 2005, Teva Pharmaceuticals returned valrocemide to Yissum, the Technology Transfer Company of the Hebrew University of Jerusalem. In June 2006, Yissum and Shire LLC entered into a worldwide license agreement whereby Shire LLC will continue the development of valrocemide. ${ }^{48}$

\section{OTHER THERAPEUTIC UTILIZATIONS OF VPA AND ITS DERIVATIVES}

Outside the domain of epilepsy, the (R)-enantiomer of a homologue of VPA, arundic acid (ONO-2506 [Ono Pharmaceuticals] or (R)-2-propyloctanoic acid), has been under clinical development for potential treatment of stroke (phase II, injectable formulation; Proglia, Ono Pharmaceuticals), as well as Alzheimer's and Parkinson's diseases (phase I, oral formulation; Cereact, Ono Pharmaceuticals). ${ }^{49}$ Unfortunately, in May 2005 the independent Data Safety Monitoring Board, after an interim analysis, recommended that ONO Pharmaceuticals discontinue its phase II clinical study of ONO-2506 (Proglia) in acute stroke in the U.S. and Canada. The analysis concluded that it is highly unlikely that injectable ONO2506 would show statistically significant efficacy compared with placebo in the current study design. Ono Pharmaceuticals is still pursuing the clinical trials of ONO-2506 (Cereact) in neurodegenerative diseases. ${ }^{49}$

The most recent new utilization of VPA as a plausible anticancer agent is unrelated to its AE and CNS activity. Both the anticancer activity of VPA and its teratogenicity were found to be connected to its ability to inhibit the enzyme histone deacetylases (HDACs). ${ }^{50}$ The SAR studies might provide needed insight into the molecular structures or the pharmacophore responsible for HDAC inhibition and consequent potent antitumor activity in a VPA analogue or derivative. ${ }^{50-52}$

The cytokine-induced maintenance and expression of the human hematopoietic stem cell population have been recently demonstrated for VPA. ${ }^{53}$ These findings underscore the potential of novel epigenetic approaches in transplantation, gene, and neurological therapies. Bug et al. ${ }^{54}$ reported that the combination of VPA with all-trans retinoic acid is effective in patients with advanced acute myeloid leukemia, leading to blast cell reduction and improvement of hemoglobin. Investigation of the effect of VPA on normal hematopoietic stem cells (HSC) shows that the drug increases both proliferation and selfrenewal of HSC. ${ }^{55}$

The SAR studies of VPA structurally related compounds clearly show that two novel VPA derivatives, 
2-pentyl-4-pentynoic acid and 2-hexyl-4-pentynoic, were found to be more potent as differentiation-inducing compounds than VPA in human myeloid leukemia cells. ${ }^{56}$ Being of equal toxicity on bone marrow cells but more active than VPA, these compounds may possibly represent a novel type of anticancer drug for the treatment of acute myeloid leukemia. ${ }^{50,56}$

Valproic acid increases fetal hemoglobin synthesis in human cell lines and in patients with epilepsy and sickle cell disease. This disease is the most prevalent of singlegene genetic diseases worldwide, associated with morbidities and mortality. ${ }^{56}$ Hydroxyurea is the only drug currently used for the treatment of patients suffering from hemoglobinopathies. ${ }^{58}$ It is believed that the effect of VPA on fetal hemoglobin synthesis is at least partly mediated by inhibition of HDACs. Analogues and derivatives of VPA are suitable candidates for designing new drugs for the treatment of $\beta$-hemoglobinopathies.

Another recent study has demonstrated that chronic VPA treatment triggers increased neuropeptide Y expression and signaling in rat nucleus reticularis thalami. ${ }^{59}$ Neuropeptide $\mathrm{Y}$ is an endogenous anticonvulsant and it has a measurable effect on synchronized neuronal activity. ${ }^{60}$ Since VPA is used in the management of absence and complex partial seizures, specific neuropeptide $\mathrm{Y}$ upregulation in rat nucleus reticularis thalami involved in the propagation of absence and temporal lobe seizures may constitute one of its clinical mechanisms of action. $^{61}$

\section{DISCUSSION AND CONCLUSIONS}

The development of new antiepileptics and CNS drugs presents economic, scientific, and medical challenges. A new AED can be successful if at least one of the following criteria are met: 1) greater efficacy in refractory epilepsy; 2) ability to prevent or delay the onset of epilepsy (epileptogenesis) and/or potential for disease modification; 3) broad use in other nonepileptic CNS disorders; 4) fewer side effects and/or better tolerability; 5) improved ease of use (namely, rapid titration, linear pharmacokinetics, lack of drug interaction, and once or twice daily dosing). Most of the new AEDs listed in Table 1 have the potential to meet some of the above criteria. If they meet the third criterion on the list, there is both a scientific and economic incentive for their development.

As previously mentioned, VPA is the "most popular" candidate for further development of second generation AEDs. ${ }^{6-8,12,13}$ Indeed, two of its amide derivates, VPD and VCD, have been marketed in some European countries for more than 35 years. ${ }^{6-8}$ However, VPD (the CNSactive corresponding amide of VPA) failed to become a second generation to VPA due to its presystemic biotransformation to VPA. ${ }^{9}$ Valnoctamide (Nirvanil, Ono
Pharmaceuticals), the corresponding amide of valnoctic acid (VCA) and a nonteratogenic constitutional isomer of VPA, has been marketed in several European countries for more than 35 years and is currently undergoing clinical trials in patients with bipolar disorder. ${ }^{34}$ Unlike VPD, VCD acts as a drug on its own and not as a prodrug to its corresponding acid VCA. The marketing of VCD in Europe was halted a few years ago, presumably due to commercial reasons. Nevertheless, the prominent CNS activity of VCD in animal models for epilepsy and neuropathic pain ${ }^{30,33}$ and its activity in patients with bipolar disorder, ${ }^{34}$ coupled with its long clinical experience in Europe as an anxiolytic drug, make VCD an attractive candidate to become a successful second-generation VPA. First, racemic VCD can be developed. Subsequently, after a chiral switch, one of its four individual stereoisomers with the best CNS activity and safety margin can be further developed. ${ }^{30}$

A pharmacokinetic-based design of nonteratogenic amide derivatives of VPA was undertaken which led to the development of valrocemide, VCD and its stereoisomers, PID and its enantiomers (R)-PID and (S)-PID, MTMCD, TMCU, and OM-TMCD (FIG. 2). These compounds have displayed promising activity in various animal models for epilepsy, neuropathic pain, and bipolar disorder. The selection of the best candidate for clinical trials can be done only after a complete toxicological package, which is mandatory for phase I clinical trials, and an investigational new drug submission. Even with their promising performances in various animal models of epilepsy and neuropathic pain and their lack of teratogenicity (in animal models), only clinical trials in refractory epileptic patients or other target patient populations can provide a definitive answer regarding the actual potential to become a new CNS drug as secondgeneration VPA.

The advantage of developing second-generation AEDs based on existing AEDs is that extensive information about pharmacokinetic, toxicological, and pharmacodynamic problems associated with the parent drug is known, and a vast array of anticonvulsant animal models have been developed that have a proven reputation and predication capabilities since the discovery of phenytoin in $1938 .{ }^{62}$ In addition, second-generation AEDs based on structural modifications of existing AEDs are less risky economically for the industry than the development of completely new chemical entities that possess new mechanisms of action.

At present, three CNS-active drugs derived from the VPA structure, valrocemide, VCD, and NPS 1776, are undergoing clinical trials. Valrocemide is in phase II clinical trials. In animal studies, it was found to be nonteratogenic and more potent than VPA. In humans, it is mainly biotransformed to valproyl glycine and only $5 \%$ of the dose is biotransformed to VPA. ${ }^{1,46-48}$ Isovaleram- 
ide is being developed as a new AED, but its pharmacokinetic profile, including its short half-life, is a drawback. Valnoctamide has recently demonstrated activity in patients with bipolar disorder. ${ }^{34}$

The present strategies for the development of new second-generation VPA drugs include novel chemical approaches for the syntheses of specific CNS-active VPA analogues and derivatives and their screening in various animal (rodent) models that have been the engines driving AED discovery and development. ${ }^{8} \mathrm{~A}$ broader and deeper understanding of the physiological role of VPA and multiple mechanisms of action will contribute to the development of new and clinically useful second-generation VPA drugs.

\section{FUTURE DIRECTIONS}

We believe that, with the utilization of second-generation VPA drugs in addition to epilepsy research, pharmacological research in the next decade will proceed (at least in part) along the following paths:

1) A new mechanism of action for VPA as an additional inducer of replication-independent active DNA demethylation in living cells, ${ }^{63}$

2) the effectiveness of VPA and its derivatives in regulating the growth differentiation and apoptosis of tumor cells;

3) the effect of second-generation tVPA drugs on the stimulation of total hemoglobin production in individuals with $\beta$-thalassemia and sickle cell diseases;

4) the role of VPA and second-generation VPA drugs in mediating the neuroprotective action of cortical neurons from spontaneous cell deaths; ${ }^{64}$

5) research into the basic neurological mechanisms of brain physiology and novel pharmacotherapies for the treatment of pain and bipolar disorder by VPA analogues and derivatives.

In the Holy Scripture it is written: "If there is not flour there is no Torah." This has meaning for academia and industry as well. To achieve commercialization, academic initiatives must present an incentive to the pharmaceutical industry. If the current clinical trials of arundic acid and ONO- $2506^{49}$ in neurodegenerative diseases and of VPA in cancer patients is successful, ${ }^{50-53}$ they may serve as models and incentives for future research that opens the way for the possible utilization of other VPA analogues and derivatives in the treatment of neuropathic pain and Parkinson's and Alzheimer's diseases. Thus, the design and development of broad-spectrum AEDs will be more attractive to industry as their utilization in epilepsy opens doors for subsequent therapeutic use (after regulatory approval) in other nonepileptic CNS disorders. ${ }^{57}$

\section{REFERENCES}

1. Bialer M, Johannessen SI, Kupferberg H, Levy RH, Perucca E, Tomson T. Progress report on new antiepileptic drugs: a summary of the seventh Eilat conference on new antiepileptic drugs (EILAT VII). Epilepsy Res 2004;61:1-48.

2. Bialer M, Walker MC, Sander JWS. Pros and cons for the development of new antiepileptic drugs. CNS Drugs 2004;16:285-289.

3. Perucca E. The new antiepileptic drugs: pharmacological and clinical aspects. Curr Pharm Des 2000;6:839-860.

4. Bialer M. New antiepileptic drugs currently in clinical trials: is there a strategy in their development? Ther Drug Monit 2002;24: 85-90.

5. Walker MC, Sander JWS. The impact of new antiepileptic drugs on the prognosis of epilepsy: seizure freedom should be the ultimate goal. Neurology 1996;46:912-914.

6. Isoherranen N, Yagen B, Bialer M. New CNS-active drugs which are second generation valproic acid: can they lead to development of the magic bullet? Curr Opin Neurol 2003;16:203-211.

7. Bialer M. New antiepileptic drugs that are second generation to existing antiepileptic drugs. Expert Opin Investig Drugs 2006;15: 637-647.

8. Rogawski MA. Diverse mechanisms of antiepileptic drugs in the development pipeline. Epilepsy Res 2006;69:273-294.

9. Perucca E. Pharmacological and therapeutic properties of valproate. A summary after 35 years of clinical experience. CNS Drugs 2002;16:695-714.

10. Loscher W. Valproate. Basel, Switzerland: Birkhauser Verlag; 1999.

11. Loscher W. Basic pharmacology of valproate. A review after 35 years of clinical use for the treatment of epilepsy. CNS Drugs 2002;16:669-694.

12. Peterson GM, Maunton M. Valproate: a simple chemical with so much to offer. J Clin Pharm Ther 2005;30:417-421.

13. Trojnar MK, Weirzchowska-Cioch E, Krzyzanowski M, Jargiello M, Czuzczwar SJ. New generation of valproic acid. Pol J Pharmacol 2004;56:283-288.

14. Nau H, Hauck R-S, Ehlers K. Valproic acid induced neural tube defects in mouse and human: aspects of chirality, alternative drug development, pharmacokinetics and possible mechanisms. Pharmacol Toxicol 1991;69:310-321.

15. Nau H, Siemens H. Differentiation between valproate-induced anticonvulsant effect, teratogenicity and hepatotoxicity. Pharm Weekbl Sci 1992;14:101-107.

16. Nau H, Hendrickx AG. Valproic acid teratogenesis. ISI Atlas Sci Pharmacol 1987;1:52-56.

17. Nau H, Loscher W. Pharmacological evaluations of various metabolites and analogs of valproic acid: teratogenic potencies in mice. Fundam Appl Toxicol 1986;6:669-676.

18. Tang W, Palaty J, Abbott FS. Time course of $\alpha$-fluorinated valproic acid in mouse brain and serum and its effect on synaptosomal $\gamma$-aminobutyric acid levels in comparison to valproic acid. J Pharmacol Exp Ther 1997;282:1163-1172.

19. Tang W, Borel AG, Fujimiya T, Abbott SF. Fluorinated analogues as mechanistic probes in valproic acid hepatotoxicity: hepatic microvesicular steatosis and glutathione status. Chem Res Toxicol 1995;8:671-682.

20. Neuman MG, Shear NH, Jacobson-Brown PM, et al. CYP2E1 mediated modulation of valproic acid-induced hepatocytotoxicity. Clin Biochem 2001;34:211-218.

21. Grillo MP, Chiellini G, Tonelli M, Benet LZ. Effect of alphafluorination of valproic acid on valproyl-S-acyl-CoA formation in vivo in rats. Drug Metab Dispos 2001;29:1210-1215.

22. Isoherranen $N$, Yagen B, Blotnik S, et al. Characterization of the anticonvulsant activity and pharmacokinetics of propylisopropyl acetamide and its enantiomers. Br J Pharmacol 2003; 138:602-613.

23. Sobol E, Bialer M, Yagen B. Tetramethylcyclopropyl analogue of a leading antiepileptic drug, valproic acid. Synthesis and evalua- 
tion of anticonvulsant activity of its amide derivatives. J Med Chem 2004;47:4316-4326.

24. Winkler I, Sobol E, Yagen B, Steinman A, Devor M, Bialer M. Efficacy of antiepileptic tetramethylcyclopropyl analogues of valproic acid amides in a rat model for neuropathic pain. Neuropharmacology 2005;49:1110-1120.

25. Morre M, Keane PE, Vernieres JC, Simiand J, Roncucci R. Valproate: recent findings and perspectives. Epilepsia 1984;25(suppl 1):S5-S9.

26. Haj-Yehia A, Bialer M. Structure-pharmacokinetic relationships in a series of valpromide isomers with antiepileptic activity. Pharm Res 1989;6:683-689.

27. Haj-Yehia A, Bialer M. Structure-pharmacokinetic relationships in a series of fatty acid amide isomers that possess anticonvulsant activity. J Pharm Sci 1990;79:719-724.

28. Badir K, Haj-Yehia A, Vree TB, van der Kleijn E, Bialer M. Pharmacokinetics and anticonvulsant activity of three mon-esteric prodrugs of valproic acid. Pharm Res 1991;8:750-753.

29. Bialer M. Clinical pharmacology of valpromide. Clin Pharmacokinet 1991;20:114-122.

30. Isoherranen N, White HS, Klein B, et al. Pharmacokinetic-pharmacodynamic relationships of $(2 \mathrm{~S}, 3 \mathrm{~S})$-valnoctamide and its stereoisomer $(2 \mathrm{R}, 3 \mathrm{~S})$-valnoctamide in rodent models of epilepsy. Pharm Res 2003;20:1293-1301.

31. Spiegelstein O, Yagen B, Levy RH, et al. Stereoselective pharmacokinetics and pharmacodynamics of propylisopropyl acetamide, a CNS-active chiral amide analog of valproic acid. Pharm Res 1999; 16:1582-1588.

32. Barel S, Yagen B, Schurig V, et al. Stereoselective pharmacokinetic analysis of valnoctamide in healthy subjects and epileptic patients. Clin Pharmacol Ther 1997;61:442-449.

33. Winkler I, Blotnik S, Shimshoni J, Yagen B, Devor M, Bialer M. Efficacy of antiepileptic isomers of valproic acid and valpromide in a rat model for neuropathic pain. Br J Pharmacol 2005;146:198208.

34. Applebaum J, Gayduk J, Agam G, Bersudsky Y, Belmaker RH. Valnoctamide as valproate substitute with low teratogenic potential: double blind controlled clinical trial. Bipolar Disord 2005;7(suppl 2):30. http://www.clinicaltrials.gov/ct/show/NCT00140179.

35. Eadie MJ. Could valerian have been the first anticonvulsant? Epilepsia 2004;45:1338-1343.

36. Bialer M, Hadad S, Kadry B, et al. Pharmacokinetic analysis and antiepileptic activity of tetramethylcyclopropyl analogues of valpromide. Pharm Res 1995;13:284-289.

37. Isoherranen N, White HS, Finnell RH, et al. Anticonvulsant profile and teratogenicity of N-methyl-tetramethylcyclopropyl carboxamide: a new antiepileptic drug. Epilepsia 2002;43:115-126.

38. Sobol E, Yagen B, White HS, et al. Preclinical evaluation of 2,2,3,3-tetramethylcyclopropanecarbonylurea, a novel second generation to valproic acid, antiepileptic drug. Neuropharmacology 2006;51:933-946.

39. Sobol E, Yagen B, Winkler I, Britzi M, Gibson D, Bialer M. Pharmacokinetics and metabolism of a new potent anticonvulsant agent 2,2,3,3-tetramethylcyclopropylcarbonylurea in rats. Drug Metab Dispos 2005;33:1538-1546.

40. Okada A, Onishi Y, Aoki K, et al. Teratology studies of derivatives of tetramethylcyclopropyl amide analogues of valproic acid in mice. Birth Defect Res (Part B) 2006;77:1-7.

41. Shaltiel G, Shamir A, Shapiro J, et al. Valproate decreases inositol biosynthesis. Biol Psychiatry 2004;56:868-874.

42. Sobol E, Yagen B, White HS, et al. Anticonvulsant activity, neural tube defect induction, mutagenicity and pharmacokinetics of a new potent antiepileptic drug, N-methoxy-2,2,3,3-tetramethylcyclopropane carboxamide. Epilepsy Res 2006 [Epub ahead of print].

43. Hadad S, Bialer M. Pharmacokinetic analysis and antiepileptic activity of N-valproyl derivatives of GABA and glycine. Pharm Res 1995;12:905-910.
44. Isoherranen N, Yagen B, Speigelstien O, et al. Anticonvulsant activity, teratogenicity and pharmacokinetics of novel valproyltaurinamide derivatives in mice. Br J Pharmacol 2003;139: $755-764$.

45. Isoherranen N, Woodhead JH, White HS, Bialer M. Anticonvulsant profile of valrocemide (TV1901): a new antiepileptic drug. Epilepsia 2001;42:831-836.

46. Hovinga CA. Valrocemide. Curr Opin Investig Drugs 2004;5:101106.

47. Bialer M, Johannessen SI, Kupferberg HJ, Levy RH, Loiseau P, Perucca E. Progress report on new antiepileptic drugs: a summary of the sixth Eilat conference on new antiepileptic drugs (EILAT VI). Epilepsy Res 2002;51:31-71.

48. Bialer M, Johannessen SI, Kupferberg HJ, Levy RH, Perucca E, Tomson T. Progress report on new antiepileptic drugs: a summary of the eighth Eilat conference on new antiepileptic drugs (EILAT VIII). Epilepsy Res (in press).

49. de Paulis T. ONO-2506. Curr Opin Investig Drugs 2003;4:863867

50. Blaheta RA, Cintal J. Anti-tumor mechanism of valproate: a novel role of an old drug. Med Res Rev 2002;22:491-511.

51. Blaheta RA, Nau H, Michaelis M, Cintal J. Valproate and valproate-analogues: potent tools to fight against cancer. Curr Med Chem 2002;9:1417-1433.

52. Eyal S, Yagen B, Shimshoni J, Bialer M. Histone deacetylases inhibition and tumor cells cytotoxicity by CNS-active VPA constitutional isomers and derivatives. Biochem Pharmacol 2005;69: 1501-1508.

53. De Felice L, Tararelli C, Mascolo MG, et al. Histone deacetylase inhibitor valproic acid enhances cytokine-induced expansion of human hematopoietic stem cells. Cancer Res 2005;65:1505-1513.

54. Bug G, Ritter M, Wassmann B, et al. Clinical trial of valproic acid and all-trans retinoic acid in patients with poor-risk acute myeloid leukemia. Cancer 2005;104:2717-2725.

55. Bug G, Gul H, Schwartz K, et al. Valproic acid stimulated proliferation and self-renewal of hematopoietic stem cells. Cancer Res 2005;65:2537-2541.

56. Deubzer H, Busche B, Ronndahl G, et al. Novel valproic acid derivatives with potent differentiation-inducing activity in myeloid leukemia cells. Leuk Res 2006;30:1167-1175.

57. Selby R, Nisbet-Brown E, Basran RK, Chang L, Olivieri NF. Valproic acid and augmentation of fetal hemoglobin in individuals with and without sickle cell disease. Blood 1997;90:891-893.

58. Atweh GF, De Simone J, Sauntharajah Y, et al. Hemoglobinopathies. Hematology Am Soc Hematol Educ Program 2003;1:14-39.

59. Brill J, Lee M, Zhao S, Fernald RD, Huguenard JR. Chronic valproic acid treatment triggers increased neuropeptide $\mathrm{Y}$ expression and signaling in rat nucleus reticularis thalami. J Neurosci 2006;26:6813-6822.

60. Sun QQ, Barban SC, Prince DA, Huegenard JR. Target-specific neuropeptide Y-ergic synaptic inhibition and its network consequences within the mammalian thalamus. J Neurosci 2003;23: 9639-9649.

61. Wilmore LJ. Divalproex and epilepsy. Psychopharmacol Bull 2003;37(suppl 2):43-53.

62. Bialer M, Twyman RE, White HS. Correlation between anticonvulsant-ED50 values of antiepileptic drugs in mice and rats and their therapeutic doses and plasma levels. Epilepsy Behav 2004; 5:866-872.

63. Detich N, Bovenzi V, Szyf M. Valproate induces replicationindependent active DNA demethylation. J Biol Chem 2003;278: $27586-27592$.

64. Jeong MR, Hashimoto R, Senatorov V, et al. Valproic acid, a mood stabilizer and anticonvulsant, protects rat cerebral cortical neurons from spontaneous cell death: a role of histone deacetylase inhibition. FEBS Lett 2003;542:74-78. 